This item was submitted to Loughborough's Research Repository by the author.

Items in Figshare are protected by copyright, with all rights reserved, unless otherwise indicated.

\title{
Liquid-liquid membrane dispersion in a stirred cell with and without controlled
} shear

PLEASE CITE THE PUBLISHED VERSION

http://dx.doi.org/10.1021/ie0504699

PUBLISHER

(c) American Chemical Society

VERSION

AM (Accepted Manuscript)

LICENCE

CC BY-NC-ND 4.0

\section{REPOSITORY RECORD}

Kosvintsev, S.R., G. Gasparini, R.G. Holdich, lain W. Cumming, and Michael T. Stillwell. 2012. "Liquid-liquid Membrane Dispersion in a Stirred Cell with and Without Controlled Shear". figshare.

https://hdl.handle.net/2134/9302. 
This item was submitted to Loughborough's Institutional Repository (https://dspace.lboro.ac.uk/) by the author and is made available under the following Creative Commons Licence conditions.

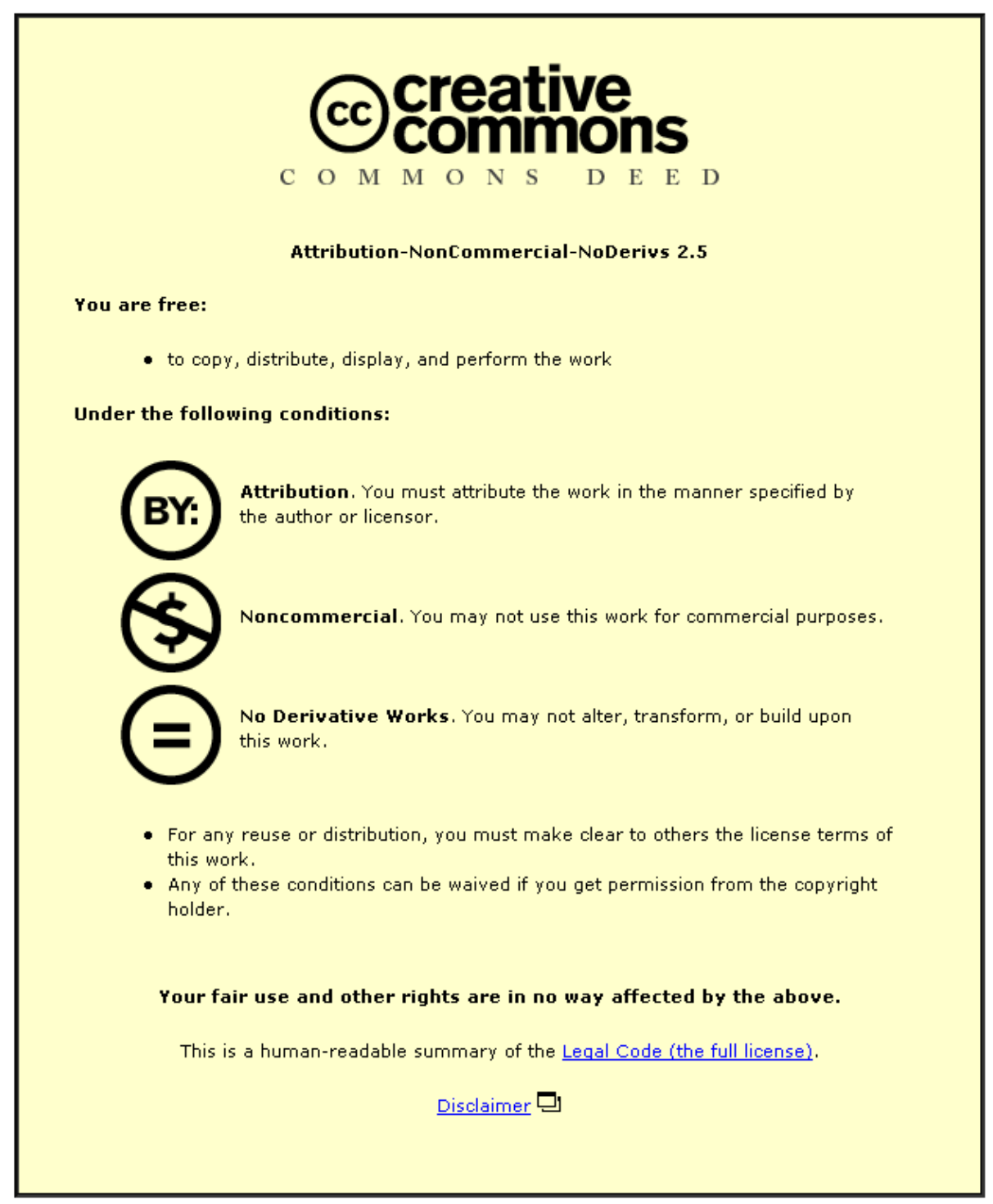

For the full text of this licence, please go to: http://creativecommons.org/licenses/by-nc-nd/2.5/ 


\title{
Liquid-Liquid membrane dispersion in a stirred cell with and without controlled shear
}

\author{
Serguei R. Kosvintsev ${ }^{1}$, Gilda Gasparini ${ }^{1}$, Richard G. Holdich ${ }^{1 *}$, Iain W. Cumming ${ }^{1}$, \\ and Michael T. Stillwell ${ }^{2}$ \\ ${ }^{1}$ Department of Chemical Engineering, Loughborough University, \\ Leicestershire, LE11 3TU, U.K. \\ ${ }^{2}$ Micropore Technologies LTD, Epinal Way, Loughborough, Leics., LE11 3 TP.
}

\begin{abstract}
Oil was passed through membranes into a continuous water phase containing a surfactant (Tween 20) to form oil dispersions with drop diameters between 40 and 400 microns. Two types of stirred equipment were used: a Weissenberg rheometer (cone and plate geometry) providing constant shear stress at all radial positions which was modified to include a membrane instead of the plate and a simple stirred cell, with a paddle rotating above the membrane, providing variable shear with radial position. Experiments show that the simple paddle stirred cell provided an oil drop dispersion that was as monosized as that produced by the controlled shear device, if not better. An analysis indicated that only the section of the membrane close to the radius of the highest shear under the paddle stirred membrane produced oil drops. The membranes used in the experiments contained a regular array of non-tortuous pores uniformly spaced and provided oil injection rates up to $1000 \mathrm{l} \mathrm{m}^{-2} \mathrm{~h}^{-1}$, which is much higher than reported fluxes for the alternative tortuous pore channel membranes made by sintering.
\end{abstract}

keywords

emulsification, rheology, sieve-membrane, force balance

*corresponding author's address:

Dr Richard Holdich, Department of Chemical Engineering, Loughborough University, Loughborough, Leicestershire, LE11 3TU, UK. Tel: +44 1509 222519, Fax: +44 1509 223923, e-mail: r.g.holdich@Lboro.ac.uk 


\section{INTRODUCTION}

Interest in the use of microfiltration membranes to produce emulsions and dispersions of one liquid phase within another immiscible liquid phase has grown considerably over the past twenty years. There exists extensive review papers covering the application of the technique to food emulsions, the industrial outlook and a recent paper discussing emulsion and particulate products based on membrane emulsification $^{1-3}$

The use of a membrane to produce an emulsion, or dispersion, is widely reported to have many advantages over alternative methods of dispersion generation such as: homogenisers, static mixers with pumps and stirred tanks. These advantages include the removal of the requirement for an emulsifying agent and the lower energy per unit volume of product required. A further important advantage is the ability to control, or engineer, the drop size together with the ability to provide a narrowly sized dispersion. An emulsifying agent may be unnecessary because the new surface of the liquid being dispersed is formed using the membrane and not by the break up of an existing surface. However, a stabilising agent is still required in order to stabilise the newly formed dispersed phase surface within the continuous phase. Hence, it is argued that an emulsifying/stabilising agent is still necessary, but the amount of that agent is less than that required to produce a dispersion by breaking larger drops. The reduction in energy requirement by using membrane emulsification is very significant ${ }^{4}$ and the ability to form near monosized dispersions in a technique that can be scaled from laboratory investigation to industrial scale application makes the process very attractive ${ }^{5}$

In most membrane emulsification investigations the intention is to produce emulsions with a dispersed phase droplet size of less than 10 microns. These would be 
appropriate for food emulsions, spacers in LCD equipment, tracer and calibration particles, etc. For the production of larger dispersion drops, between 10 and 250 microns, membrane processes have still been suggested, but there is less work published and the degree to which the drop size distribution can be described as monosized usually becomes less acceptable. This is a size range of commercial interest as particles can be formed from the polymerisation, or solidification by some means, of the liquid that forms the dispersed phase. Such particles are of use for monosized column packings, medical diagnostic strips, functionalised beads and as encapsulated particles with an internal phase containing a controlled release compound. In the latter case, there is often the need for the preparation of a primary emulsion which is then dispersed to form a secondary emulsion. This could be an internal water phase encapsulated in an oil phase and then dispersed into another water phase $(\mathrm{w} / \mathrm{o} / \mathrm{w})$, or an inner oil core encapsulated by a shell formed in the aqueous phase and then dispersed into an oil phase (o/w/o).

The production of monosized dispersions with drop sizes greater than 10 microns has some additional challenges when compared to the production of fine monosized drops. Primarily, the need to keep the inherently more unstable drops in such a way as to prevent them coalescing, or breaking up in a shear field associated with the membrane process. Thus, crossflow membrane emulsification is less likely to be successful with large drops, due to the need to pump the continuous phase around a flow circuit. In this case, the less stable drops could break up in the shear of the pump, or on passage through the fittings around the flow loop. To overcome this problem it is possible to use a rotating membrane system ${ }^{6}$, but there is little published operating data on such a system. Data has been published on a simple stirred system in which a circular flat sheet membrane was used, through which salt water was injected onto a continuous phase of kerosene and soybean oil. The shear at the membrane surface was 
provided by a simple magnetic stirrer ${ }^{7}$. A reasonably monosized dispersion was obtained, despite the obviously inhomogeneous shear field generated by the magnetic stirrer. The drop size produced in the study was 5 to 10 microns, using membrane pores sizes of nominally 1 and 5 microns. However, there was no consideration of the effect of shear, just an investigation of drop size and its variation with membrane pore size in the stirred system.

The complexity of generation of larger drops sizes has led to a claim that there is no known membrane technique for their reliable manufacture ${ }^{8}$, due to the pore size distribution of the membrane. In this case, the authors considered only the availability of the tortuous pore channel microfiltration membranes formed by sintering and did not consider the availability of the flat lamina membranes, without tortuous pore channels, that is used in the current study. Thus, an alternative approach to membrane emulsification was described based on several microchannels operating in parallel ${ }^{\mathbf{8}}$. These are essentially several capillaries etched into a silicon surface that provide large dispersed phase liquid drops that spontaneously snap-off on injection into the continuous phase due to the surface tension of the forming drop and the irregular channel shape through which they are passed.

In most cases of membrane emulsification, crossflow of the continuous phase over the surface of the membrane is employed. When using a tubular geometry it is reasonable to assume that this provides a uniform and consistent shear stress at the membrane wall. Thus, if the shear is consistent it is argued that the drops detached from the surface of the membrane will be of a consistent size. In most modelling a balance of forces is employed, in which the force resisting detachment is the capillary force and the forces causing drop detachment are a shear/drag force and a buoyancy force (for upward facing flat membranes with drops that float). However, in most cases the 
buoyancy force is negligible. When using a flat disc type membrane it is possible to generate a uniform shear field using the same principle as the cone and plate rheometer, such as a Weissenberg rheometer. The following experimental study reports the adaptation of a Weissenberg rheometer for use as a means of dispersion generation and compares the resulting drop size distribution from such a highly controlled shear device with a simple paddle bladed stirred cell used to produce the same dispersion. Modelling of the process is achieved by a force balance and by a torque balance, both modified for the stirred system under consideration.

An interesting alternative modelling approach to the simple force balance has been proposed ${ }^{9}$ in which the Gibbs free energy is considered using an open-source finite element package known as Surface Evolver. The drop size predicted by the model is based on what was termed the Pressure Pinch Constraint. This occurs when the Laplace pressure of the remaining segment of liquid attached to the pore is equal to the pressure within the drop. The latter is calculated from the total energy, area and volume outputs from the Surface Evolver software. The solution was found to be highly dependent on the geometry of the pore. However, this approach is only valid for systems in which there is no contribution towards drop detachment from effects due to: inertia, gravity, kinetic energy or a shear component within the continuous phase. For systems employing shear as a mechanism for drop removal from the membrane surface, either a force balance or torque balance approach is still required.

From a practical point, one of the main reported problems with membrane emulsification using a sinter type membrane is the low productivity of the process. When producing dispersions using a 15 micron pore diameter membrane providing drop sizes of soybean oil of up to 40 microns dispersed in surfactant containing water, the maximum oil flux through the membrane was reported as 20 litres of oil per 
square metre of membrane area per hour $\left(20 \mathrm{l} \mathrm{m}^{-2} \mathrm{~h}^{-1}\right){ }^{\mathbf{1 0}}$. However, to obtain a narrow drop size distribution the process had to be operated at a flux rate one half of this value. In order to increase the oil flux rate to values that may well be commercially viable the technique of forming a pre-mix emulsion, which has a poor size distribution, which is then put through the membrane to improve the size distribution has been described ${ }^{\mathbf{1 1}}$. The pre-mix membrane emulsification process is reported to have high oil flux rates, but requires multiple passes through the membrane matrix. Hence, the commercial advantage of the technique is reduced. Another disadvantage of the pre-mix process is the capacity for fouling during the process, which with the tortuous pore channel sinter membranes can be severe due to membrane depth fouling $^{12}$. This becomes a more serious problem with the use of the membranes for multiple emulsions, where there is two phase flow within the matrix of the sinter membrane. For both multiple and single emulsions the design of the membrane is important and there would be practical advantages in avoiding the use of the sinter type of membrane.

The sinter type of porous membrane is typically made from glass, or ceramic, material. These are available for microfiltration use with equivalent pore sizes of between 0.1 and 10 microns. They are usually tortuous microfilters, that consist of a network of flow channels made by a variety of means. They do not have uniform diameter holes passing though them and the surface pore openings are very irregular. However, it is assumed that the pressure at which a bubble will emanate from the surface when the filter is submerged in a fully wetting fluid is equal to the capillary pressure of a perfectly round pore. Hence the term 'equivalent', or 'nominal' pore size. It is usual for there to be a substantial pore size distribution with this type of membrane and the pore openings are far from circular. Thus, there is evidence that only a very small fraction of the available pores are used in the process of dispersion 
formation. This will be the larger tortuous pores, that will take most of the flow and the percentage of total pores actively producing dispersion may well be less than $1 \%^{\mathbf{1 0}}$. In contrast to the conventional sintered membranes, the work described here used a newly available membrane with a regular array of uniform pores, see Figure 1. Here the pore geometry, spacing and size are very well defined and can be measured for the purposes of modelling and analysis. Similar membranes with an array of very well defined pores have been used previously, but in crossflow emulsification and with pores closely spaced together leading to coalescence on the surface of the membrane during drop formation ${ }^{\mathbf{1 3}}$. This led to a wide size distribution from the membrane emulsification process. Further work was reported into modelling the process by CFD in order to determine the optimum spacing of the pores on a membrane, taking into account the deforming drops on the surface of the membrane prior to detachment and the need to avoid coalescence between the drops ${ }^{\mathbf{1 4}}$. However, to-date there have been no reports of the computational findings from this work being experimentally validated. Nevertheless, the important conclusion from the work was that unlike in conventional microfiltration the ideal membrane for emulsification, or dispersion, generation requires a low density of pores per unit area. The optimum surface porosity of an emulsification membrane was found to be significantly less than $5 \%$ of the total surface area.

\section{Drop size distribution assessment}

The degree to which a particle, or drop, size distribution can be described as monosized can be indicated by several different measurements. The simplest one is to calculate the span, which is defined as the particle diameter which $90 \%$ of the distrtibution lies below $\left(x_{0.9}\right)$ minus the diameter which $10 \%$ of the distribution lies below $\left(x_{0.1}\right)$ divided by the median diameter of the distribution $\left(x_{0.5}\right)$, i.e. 
Span $=\frac{x_{0.9}-x_{0.1}}{x_{0.5}}$

In general, material with a span significantly less than unity can be described as monosized.

\section{Dispersed drop size modelling}

The following analysis follows a conventional force, and torque balance, approach but adopted to shear in a stirred cell. Figure 2 illustrates two possible deforming states for an immiscible drop injected into a liquid continuous phase: a weakly deformed drop in which the drop may be assumed to remain spherical and here the most appropriate mechanical model of drop detachment would be a torque balance, and a strongly deformed drop where a simple force balance at the pore exit would be the most suitable model. It is possible that a practical system could lie between these two extremes. Both the torque and force balances can be used to derive equations when the torque, or force, is in balance, which will define the limiting size of the drop before detachment occurs. Hence, the two different models should predict the size of the drops formed.

A simplified form of the torque balance, where buoyancy and lift forces have been neglected is

$$
M_{c z}=F_{s} \frac{x}{2} \cos (\alpha)
$$

where the projection of the capillary force, or moment, onto the axis of the rolling drop is $M_{c z}, \alpha$ is the angle from the pore edge, $x$ is the drop diameter and $F_{s}$ is the force due to the shear at the surface. The moment arising from the capillary force can be obtained by integration of the vector product $\vec{M}_{c}=\oint_{L} \vec{r} \otimes \mathrm{d} \vec{F}_{c}$, where the integration takes place along the pore perimeter $L$. Here $\mathrm{d} \vec{F}_{c}$ is the force acting on 
unit length of the contact line. The force has magnitude $\gamma \mathrm{d} L$ and is directed towards the pore. In order to find the projection of the force to a moment the vector integration becomes a scalar

$$
M_{c z}=2 \int_{0}^{2 r_{p}} \gamma r \mathrm{~d} r=2 \pi r_{p}^{2} \gamma
$$

where $\gamma$ is the interfacial tension and $r_{p}$ is the pore radius. The shear force can be estimated from an approach based on Stokes' drag, but modified for the nearness of a wall and assuming that the shear flow velocity for a Newtonian liquid can be deduced from Newton's law of viscosity and the geometry of the drop sitting on a pore entrance (see Figure 2). Equating equations (1) and (2) using Stokes' drag expression for the shear force, with a constant that may be taken to be equal to 3 to account for the closeness of the wall ${ }^{15}$, and assuming Newtonian flow near the surface the following equation for shear force results

$$
F_{s}=9 \pi \tau x \sqrt{\left(\frac{x}{2}\right)^{2}-r_{p}^{2}}
$$

Substituting equations (2) and (3) into equation (1) and eliminating the sine and cosines provides the following equation for the drop size at which detachment occurs

$$
\bar{x}=\frac{12^{1 / 3}\left(r_{p}^{2} \tau^{2}\left(\gamma+\sqrt{\gamma^{2}-12 r_{p}^{2} \tau^{2}}\right)\right)^{1 / 3}}{3 \tau}+\frac{12^{2 / 3} r_{p}^{2} \tau}{3\left(r_{p}^{2} \tau^{2}\left(\gamma+\sqrt{\gamma^{2}-12 r_{p}^{2} \tau^{2}}\right)\right)^{1 / 3}}
$$

For low values of shear stress, it can be shown that the proportionality approximates to an inverse cubic root of shear stress $x \approx \sqrt[3]{\frac{12}{27}} \sqrt[3]{\frac{\gamma}{\tau}} \sqrt[3]{r_{p}^{2}}$. At very high shear stresses it asymptotically approaches $x_{\min }=2 r_{p}$ (i.e. the pore diameter). Equation (4) can be solved, provided that the pore size is known, and the shear stress and/or rate close to the pore opening can be measured, or predicted. This is the case during the experiments using the modified Weissenberg rheometer. 
Using the alternative approach, based on a simple force balance at the pore, where

$$
F_{c}=F_{s}
$$

the resulting equation for the drop size at detachment is

$$
x=\frac{\sqrt{18 \tau^{2} r_{p}^{2}+2 \sqrt{81 \tau^{4} r_{p}^{4}+4 r_{p}^{2} \tau^{2} \gamma^{2}}}}{3 \tau}
$$

Here the asymptote for high shear stress is $x_{\min }=2 r_{p}$ again, though for small shear stress the dependency becomes $x=\frac{2}{3} \sqrt{\frac{\gamma}{\tau}} \sqrt{r_{p}}$

In a Weissenberg rheometer shear is not a function of radial position because the gap height increase compensates for the increased tangential velocity experienced by the outer radii. Under these conditions, the appropriate equations for drop size modelling are (4) and (6) above, with constant shear. However, for a simple stirred system, using a paddle blade mixer further consideration of the shear, and its variation with radial position, is required.

The approach to the shear field distribution along a paddle blade radius of Ciofalo was used ${ }^{\mathbf{1 6}}$ in the modelling. The flow field used in the stirred cell experiments is illustrated in Figure 3. Here, the tangential component of velocity under the impeller paddles is presented, rotating with angular velocity $w$. The flow field in an unbaffled cylindrical vessel can be subdivided into an inner region $r<r_{c}$ (forced vortex) which exhibits a rigid-body motion with angular velocity $w$ of the impeller, and an outer region $r>r_{c}$ (free vortex), in which angular momentum $\left(r u_{t}\right)$ is a constant ${ }^{17}$. The shear field is at a maximum at the critical radius, which is the point at which the rotation changes from a forced vortex to a free vortex. 
For flat blade paddle impellers the solution follows Yamamoto's method, as reported by Nagata ${ }^{18}$, the following experimentally found correlation can be used for the critical radius where the shear is at a maximum $r_{c}$

$$
r_{c}=\frac{D}{2} 1.23\left(0.57+0.35 \frac{D}{T}\right)\left(\frac{b}{T}\right)^{0.036} n_{b}^{0.116} \frac{\mathrm{Re}}{1000+1.43 \mathrm{Re}}
$$

Here $b$ is the blade height and $n_{b}$ is the number of blades, and Reynolds Number is defined by $\operatorname{Re}=\frac{\rho w D^{2}}{2 \pi \eta}$.

In Figure 3 the cell geometry is illustrated. For the experimental cell used $(H=12 \mathrm{~cm}$, $D=2.4 \mathrm{~cm}, n_{b}=2, T=3.5 \mathrm{~cm}$ and $\left.b=1.2 \mathrm{~cm}\right)$.

The size of the solid body rotation vortex increases with rotational speed and for a speed of $20 \mathrm{rad} / \mathrm{s}$ it occupies half the length of the blade, a free vortex exists beyond this. At $120 \mathrm{rad} / \mathrm{s}$ almost $80 \%$ of the cell is occupied by the forced vortex. For the purposes of correlating experimental data with shear stress, the maximum shear stress was used (at $r_{c}$ ) in the drop size prediction modelling.

To calculate the shear stress at the membrane surface it is assumed that the shear in the boundary layer drops down to zero at the membrane surface over a thickness $\delta$ defined by the Landau-Lifshitz ${ }^{19}$ equation

$$
\delta=\sqrt{\frac{\eta}{\rho w}}
$$

Hence shear is constant with respect to height, but not with radial position. The smallness of the drops with respect to the thickness of the boundary layer is another assumption used in the theoretical estimations. On the basis of these assumptions the shear stress at the membrane surface is

$$
\tau=0.825 \eta w r \frac{1}{\delta} \quad r<r_{c}
$$


$\tau=0.825 \eta w r_{c}\left(\frac{r_{c}}{r}\right)^{0.6} \frac{1}{\delta} \quad r>r_{c}$

Here $r_{c}$ is defined by equation (7). Equation (9b) is not strictly valid at the wall surface as it does not match the no-slip boundary condition, but the following analysis is based at radial position $r_{c}$, at which position equation (9b) reduces to (9a).

Considering drop formation at the membrane pores, it is well known that to start a drop growing the transmembrane pressure should be higher than the capillary pressure $P_{c}=\frac{2 \gamma \cos (\theta)}{r_{p}}$. When transmembrane pressure is above the capillary pressure the drops grow evenly in different places on the membrane, proportional to the difference of the pressure on the opposite sides of the drop $\Delta P=P-P_{v}-\frac{4 \gamma}{D}>P_{c}$, where $P_{v}$ is the pressure necessary for flow of the dispersed phase in the pores. After the diameter of the drop becomes bigger then $2 r_{p}$, the drops approach the critical and then, detach from the pores. It is postulated that drops located around $r=r_{c}$ come off more easily than drops formed at the membrane centre and periphery. After a drop breaks off, a nearly flat interface between the liquids at the tip of the pore forms. The pressure in those pores becomes bigger $\Delta P=P_{v}-0$, hence flux is directed into these prevailing pores. Hence, at the some relatively small value of transmembrane pressure (slightly higher then the capillary pressure) only part of the pores are active and this is around the radius with the biggest shear stress, i.e. with radius $r_{c}$. Finally, dynamic equilibrium establishes when drops come off predominantly from the place where the shear stress is at a maximum (see Figure 3). Experimental confirmation of this described effect follows in the Results section. 


\section{EXPERIMENTAL}

Two types of equipment were used to generate the dispersions: a modified Weissenberg rheometer employing a cone and plate head in contact with the continuous phase, usually used for rheological study, and a stirred cell fitted with a simple paddle stirrer driven by a $24 \mathrm{~V}$ DC motor. The modified rheometer was used for stirred experiments with consistent shear across the membrane surface and in-situ monitoring of the local viscosity during the formation of the drops at the membrane surface. In the stirred cell the shear at the membrane surface varied with radial position under the paddle blade stirrer, as described in the previous section. The speed of rotation of the paddle was varied by adjusting the voltage applied to the DC stirrer motor.

A Weissenberg 19 Rheometer was adapted so that the normally impervious plate underneath the cone was replaced by the membrane illustrated in Figure 4. A feed chamber was constructed to permit the addition of oil from underneath the membrane. A rotating seal was required; so that it was possible to rotate the base (including the membrane) underneath the cone. A chamber above the cone section was filled with continuous phase into which the oil was injected through the dividing membrane. The oil drops, after formation at the membrane surface, floated away from the cone, but the process is a batch one in which the concentration of dispersed oil drops increased. Thus, the viscosity of the dispersion increased with time during an experiment. The viscosity was measured in-situ from the Weissenberg control equipment, which determined viscosity from the torque required to keep the cone stationary within the chamber. A hydrophilic membrane with an average pore size $18 \mu \mathrm{m}$ and diameter 40 $\mathrm{mm}$ was fixed on the rotating platform in the specially designed membrane holder. The shear rate range used was $500-3000 \mathrm{~s}^{-1}$ and measured shear stresses were between 10 to $40 \mathrm{dyne} / \mathrm{cm}^{2}$. The injection rate was in the range $0.01-10 \mathrm{ml} / \mathrm{min}$ 
using a low-pulsation peristaltic pump, injected into mixtures of water with glycerol and Tween 20. The total capacity of the chamber was $150 \mathrm{ml}$ and $10-15 \mathrm{ml}$ of dispersed phase, i.e. sunflower oil, was injected into $100 \mathrm{ml}$ of continuous phase.

The paddle bladed stirred cell was supplied by Micropore Technologies Ltd and employed a paddle blade of $24 \mathrm{~mm}$ diameter, $12 \mathrm{~mm}$ above a similar hydrophilic membranes with 9,18 , or $40 \mu \mathrm{m}$ pore sizes. These membranes were supplied by Micropore Technologies Ltd. and are available commercially. They are produced by galvanic deposition of nickel onto a template formed by a photolithographic technique. The diameter of the membrane used was $34 \mathrm{~mm}$, which is the internal diameter of the glass chamber. The total glass chamber height was $155 \mathrm{~mm}$ and the normal working height of produced dispersion in the chamber was $100 \mathrm{~mm}$ providing a volume of $150 \mathrm{ml}$ of continuous phase into which $40 \mathrm{ml}$ of sunflower oil was injected at a rate between 0.5 to $3 \mathrm{ml} / \mathrm{min}$ for the $9 \mu \mathrm{m}$ membrane and 0.5 to 20 $\mathrm{ml} / \mathrm{min}$ for the $40 \mu \mathrm{m}$ pore size membrane.

In both sets of tests the dispersed phase was a food grade sunflower oil and the continuous phase consisted of water (from a reverse osmosis system), with $2 \%$ of Tween 20 (polyoxylethylene sorbitan monolaureate) provided by Sigma Aldrich and varying amounts of Polyvinyl alcohol (Fluka) and glycerol (Fisher). The PVA and glycerol were added as viscosity modifiers, but tests were independently performed to evaluate the influence of the modifiers on the interfacial tension between the aqueous and oil phases.

For evaluation of drop size distribution a Malvern Mastersizer S was used. For interfacial tension measurements a Wilhelmy ring device, employing a pull-off platinum-iridium ring with digital balance (model DB2kS) was used. A number of 
repeat experiments were performed and the mean average interfacial tension between the sunflower oil and water containing Tween 20 was $6.0 \mathrm{mN} \mathrm{m}^{-1}$, with a relative standard deviation of $17 \%$.

\section{RESULTS AND DISCUSSION}

In the initial controlled shear experiments performed using the Weissenberg rheometer the shear stress was measured whilst injecting sunflower oil into water and water/glycerol mixtures at different shear rates. These experiments were performed under conditions of consistent shear at all radial positions. The results are presented in Tables 1 and 2. Similar shear rates were used for the tests reported in Tables 1 and 2, the only difference was with the amount of glycerol employed and, therefore, the starting viscosity of the continuous phase. During injection of the oil the viscosity increased, due to the increasing concentration of dispersed phase. The starting and ending shear stress, for the fixed shear rate, is provided in both tables. The modelling equations require a fixed value of the coefficient of dynamic viscosity. Hence, as the viscosity increases due to increasing dispersed phase content and the viscosity was measured in-situ, it is possible to calculate the predicted drop size from both the starting and ending viscosity values. Thus, a predicted size range is reported in the tables using the starting and ending viscosity values in the models for the torque and force balances. This assumes that the dispersion layer between the cone and plate can be treated as a continuum, despite the two phase nature of the process and the relatively large drop size for the narrow gap employed. It is noticeable that the experimentally produced values for the median drop size lie between the predicted values for the torque and force balance approaches. It appears that the torque balance is a closest match to the data for the higher viscosity system, Table 2 . However, the force balance is a slightly better match to the data for the lower viscosity system, Table 1. It is also noticeable that the span for the distributions vary between 0.55 and 
1.4. Spans of less than unity may be deemed to represent a monosized distribution, but with such a carefully controlled shear field as given by the rheometer spans much less than unity might be expected. Furthermore, in Figure 5 there is a direct comparison between the spans achieved using the two different devices for the production of drops with median diameters between 60 and 105 microns, using the same membrane type and experimental conditions. A lower span, more monosized distribution, is provided by the paddle stirred device.

The remaining experiments refer to the results obtained from the simple stirred cell, employing a paddle blade stirrer with no attempt to control the shear such as by increasing the gap size with radial position. Further investigation of the force balance model was performed by comparing the results from the model and experiment for three different pore size membranes: 40, 18 and 9 micron diameters. The comparison involved the production of dispersion drops at a variety of different paddle rotational speeds, with measurement of the resulting size distribution by Malvern Mastersizer. The measured median sizes with respect to stirrer speed are plotted in Figure 6 as the data points and the predicted values are shown as the corresponding curves. The force balance model is in broad agreement with the experimental data, with a notably better fit at higher stirrer speeds.

The hypothesis that most of the oil drop generation takes place at a radius on the membrane that is near to the critical radius of the paddle was tested by using an impervious glue to inactivate various areas of a 18 micron pore diameter membrane. Figure 7 shows the resulting drop size distributions, obtained using the whole active membrane, a membrane inactivated to a radius of $5 \mathrm{~mm}$, to a radius of $7.5 \mathrm{~mm}$ and another membrane with both a central radius of $5 \mathrm{~mm}$ and an outer peripheral ring 5 $\mathrm{mm}$ inactivated. The drop size distribution appears to be independent of the physically inactivated membrane region. Thus, it appears reasonable to suggest that the paddle 
stirred system is self-limited in terms of the active region producing an oil dispersion - in accordance with the discussion in the dispersed drop size modelling section. During all of these experiments the injection pressure was kept at a value just slightly greater than the capillary pressure of the membrane pores.

In Figure 8 the oil throughput is shown for the 18 micron pore diameter membrane, whilst the paddle stirrer speed was maintained at $500 \mathrm{rpm}$ for all the tests. The figure includes data on the $x_{0.1}, x_{0.5}$ and $x_{0.9}$ values to enable the span to be calculated according to the earlier description of drop size distribution assessment. The first observation on the data is that the oil flux rates are up to an order of magnitude greater than what is normally achieved when using a tortuous pore channel membrane for dispersion generation ${ }^{\mathbf{1 0}}$. This is attributable to the structure of the lamina membrane, with straight-through and uniform pores, used in this study, see Figure 1. Thus, it is likely that the number of pores generating dispersion will be higher than is the case when using a membrane with a significant pore size distribution, where less than $1 \%$ of the pores may be generating dispersion ${ }^{\mathbf{1 0}}$. Also, the flow resistance of a thin lamina straight-through pore channel membrane will be much less than a tortuous pore channel membrane.

Using the data included on the figure it is possible to deduce that the span for these distributions is approximately 0.5 and does not change significantly with injection rate. The median drop size significantly increases with injection rate, but the size distribution does not appear to broaden excessively over the operating conditions illustrated. Clearly, an increasing drop size with injection rate, or transmembrane pressure drop, is not accounted for in equations (4) or (6) and these models should be regarded as providing the smallest drop size where the size is determined by the shear-capillary forces only. 
Another important observation regarding the span is that for all the reported tests the degree to which the distribution can be described as monosized is considerably better than the data obtained from the controlled shear tests using the modified Weissenberg rheometer. The best values of span obtained with the Weissenberg rheometer were 0.55 and 0.58 , over a similar median drop size range. However, with the simple stirred cell tests spans below 0.5 appear to be possible. One final observation on the nature of the membrane used in this study is that it would appear to be ideally suited to multiple dispersion production, either o/w/o or w/o/w, as the lack of an internal structure and tortuous pore channel provides no internal structure for material to become deposited within the membrane. Also, the minimal flow resistance offered by this membrane helps in the safe passage of the primary emulsion through the membrane, minimising losses due to rupture of the primary emulsion within the membrane.

\section{CONCLUSIONS}

The production of an oil dispersion with controlled average drop sizes between 40 and 400 microns has been shown to be possible using a simple paddle bladed stirred cell, without the need for controlled shear. In terms of span of the resulting drop size distribution, the simple stirred cell provided a more monosized product than the modified Weissenberg Rheometer, despite the more consistent shear conditions over the membrane surface provided by the latter device. A simple low-volume stirred cell apparatus is useful for the purpose of testing the chemistry of a proposed dispersion, i.e. surfactant type and concentration, membrane type, shear conditions, etc., For larger-scale production other techniques for the generation of the low shear values required for drop detachment are possible, as are employed in conventional industrialscale microfiltration. However, it is unlikely that crossflow dispersion generation would be appropriate, due to the destructive shear fields generated within the pumps and fittings. 
The non-tortuous pore channel membrane, with a very uniform pore size and spacing, is shown to provide dispersions with span values of less than 0.5 , whilst producing drops with median diameters greater than 40 microns. Hence, it is possible to use a membrane technique for the production of monosized drops over a wide range of drop sizes. The productivity, in terms of oil flux through the membrane, did decrease with pore size. However, for the 9 micron pore diameter membrane flux rates of up to 1801 $\mathrm{m}^{-2} \mathrm{~h}^{-1}$ were possible, without the loss of apparent degree of drop size uniformity. For the 18 micron pore size membrane oil flux rates of up to $900 \mathrm{l} \mathrm{m}^{-2} \mathrm{~h}^{-1}$, without the loss of apparent degree of drop size uniformity, were possible. These are oil flux rates of about one order of magnitude greater than is reported for tortuous pore channel membranes of the sinter type. The high fluxes are most likely attributable to the very uniform pore size and lamina nature of the membrane.

For the purpose of modelling the drop size produced with shear at the membrane surface, the simple force balance model was shown to be adequate for the stirred cell, modified for maximum shear obtained in the stirred system. The torque balance approach was reasonable for low values of shear used in the controlled shear experiments, conducted on the modified Weissenberg rheometer. This device provided a useful experimental tool for investigation of conditions during dispersion formation as it measured the viscosity of the system during the dispersion generation process. However, in terms of a more monosized oil drop product, there did not appear to be any advantage in using the modified rheometer compared to the simple paddle bladed stirred cell. It appears that in the paddle blade cell the region in which oil dispersion is generated is limited to that close to the position of maximum shear under the paddle blade. Thus, the regions where the shear is much lower do not tend to generate oil drops - hence the drop size distribution of product is much better than 
would be expected from such a non-controlled shear device. Experimental evidence from tests in which limited areas of the circular disc membrane were inactivated for oil injection confirmed this conclusion.

\section{ACKNOWLEDGEMENT}

The authors wish to thank the Engineering and Physical Sciences Research Council of the UK for an award (GR/S04758/01) which partially supported the work described here. 


\section{REFERENCES}

(1) Vladisavljevic, G.T. and Williams, R.A., Recent developments in manufacturing emulsions and particlulate products using membranes, $A d v$. Coll. \& Int. Sci., 2005, 113, 1.

(2) Joscelyne, S.M. and Tragardh, G., Membrane emulsification - a literature review, Jnl. Mem. Sci., 2000, 169, 107.

(3) Gijsbertsen-Abrahamse, A.J., van der Padt, A. and Boom, R.M., Status of cross-flow membrane emulsification and outlook for industrial application, Jnl. Mem. Sci.,, 2004, 230, 149.

(4) Peng, S.J. and Williams, R.A., Controlled production of emulsions using a crossflow membrane Part I: droplet formation from a single pore, Trans. IChemE., 1998, 76, 894.

(5) Williams, R.A., Peng, S.J., Wheeler, D.A., Morley, N.C., Taylor, D., Whalley, M. and Houldsworth, D.W., Controlled production of emulsions using a crossflow membrane Part II: industrial scale manufacture, Trans. IChemE., 1998, 76, 902.

(6) Williams, R.A., 2001, Controlled dispersion using a spinning membrane reactor, UK Patent Application No. PCT/GB00/04917.

(7) Yamazaki, N., Naganuma, K., Nagai, M., Ma, G.H. and Omi, S., Preparation of W/O (Water-in-Oil) emulsions using a PTFE (polytetrafluoroethylene) membrane - a new emulsification device, Jnl. Dispersion Sci. \& Tech., 2003, 24, 249.

(8) Sugiura, S. Nakajima, M. and Seki, M., Preparation of monodispersed polymeric microspheres over $50 \mu \mathrm{m}$ employing microchannel emulsification, Ind. Eng. Chem. Res., 2002, 41, 4043. 
(9) Rayner, M., Tragardh, G., Tragardh, C. and Dejmek, P., Using the Surface Evolver to model droplet formation processes in membrane emulsification, Jnl Coll \& Int. Sci., 2004, 279, 175.

(10) Yasuno, M., Nakajima, M., Iwamoto, S., Maruyama, T., Sugiura, S., Kobayashi, I., Shono, A. and Satoh, K., Visualization and characterization of SPG membrane emulsification, Jnl. Mem. Sci., 2002, 210, 29.

(11) Vladisavljevic, G.T., Shimidzu, M. and Nakashima, T., Preparation of monodisperse multiple emulsions at high production rates by multi-stage premix membrane emulsification, Jnl. Mem. Sci., 2004, 244, 97.

(12) van der Graaf, S., Schroen, C.G.P.H. and Boom, R.M., Preparation of double emulsions by membrane emulsification - a review, Jnl. Mem. Sci., 2005, 251,7 .

(13) Abrahamse, A.J., van Lierop, R.G.M., van der Sman, A., van der Padt, A., and Boom, R.M., Analysis of droplet formation and interactions during cross-flow membrane emulsification, Jnl. Mem. Sci., 2002, 204, 125.

(14) Abrahamse, A.J., van der Padt, A., Boom, R.M. and de Heij, W.B.C., Process fundamentals of membrane emulsification: simulation with CFD, AIChE J., 2001, 47, 1285.

(15) Keh, H.J. and Chen, P.Y., Slow motion of a droplet between two parallel plane walls, Chem. Eng. Sc., 2001, 56, 6863.

(16) Ciofalo, M., Brucato, A., Grisafi, F. and Torrata, N., Turbulent flow in closed and free-surface unbaffled tanks stirred by radial impellers, Chem. Eng. Sci., 1996, 51, 3557.

(17) Smit, L. and During, J., Vortex geometry in stirred vessels. Proceedings of the $7^{\text {th }}$ Euroean Congress on Mixing, Vol. 2, 1991, 633-639, Bruges, Belgium. 
(18) Nagata, S., Mixing: Principle and Applications, 1975, Wiley, New York.

(19) Landau, L.D. and Lifshitz, E.M., Fluid Mechanics, 1959, Pergamon Press, Oxford, UK. 
List of Tables

Table 1 Median sunflower oil drop size as a function of shear stress using the modified Weissenberg rheometer at low continuous phase viscosity

Table 2 Median sunflower oil drop size as a function of shear stress using the modified Weissenberg rheometer at elevated continuous phase viscosity 
List of Figures

Figure $1 \quad$ Regular pore array of a membrane used to produce dispersions

Figure 2 a) a weakly deformed droplet forming at the surface of a pore - leading to a torque balance at the centre of the drop and b) a strongly deformed droplet - leading to a force balance at the membrane surface.

Figure 3 a) Schematic of simple paddle blade stirred cell and

b) tangential velocity component under the impeller

Figure 4 Modified Weissenberg rheometer used for injection of oil in to aqueous phase

Figure 5 Comparison of drop size distribution spans produced using the two different devices for dispersion generation: the simple paddle blade stirred cell and the converted controlled shear rheometer

Figure 6 Drop size against rotation speed for three different membranes sizes sunflower oil in water and $1 \%$ Tween 20 solution in the simple paddle blade cell: markers are data and curves are from equation (6)

Figure 7 Drop size distributions for sunflower oil in water and $1 \%$ Tween 20 solution in the small stirred cell using a $18 \mu \mathrm{m}$ membrane with different areas inactive with rotational speed $500 \mathrm{rpm}$

Figure 8 Median drop sizes together with 10 and 90 percentile for sunflower oil in water and $1 \%$ Tween 20 solution in the small paddle blade stirred cell using an $18 \mu \mathrm{m}$ membrane with various oil injection rates at a rotational speed $500 \mathrm{rpm}$ 
Table $1 \quad$ Median sunflower oil drop size as a function of shear stress using the modified Weissenberg rheometer at low continuous phase viscosity

\begin{tabular}{|c|c|c|c|c|c|c|c|}
\hline $\begin{array}{l}\text { Shear } \\
\text { rate }\end{array}$ & $\begin{array}{l}\text { Shear stress } \\
\text { at start of } \\
\text { experiment } \\
\left(\text { dyne } / \mathrm{cm}^{2}\right) \\
\end{array}$ & $\begin{array}{l}\text { Shear stress } \\
\text { at end of } \\
\text { experiment } \\
\left(\text { dyne } / \mathrm{cm}^{2}\right) \\
\end{array}$ & $\begin{array}{c}\text { Measured } \\
\text { median } \\
\text { diameter } \\
(\mu \mathrm{m}) \\
\end{array}$ & $\begin{array}{c}\text { Span of } \\
\text { distribution }\end{array}$ & $\begin{array}{l}\text { Coefficient of } \\
\text { variation } \\
(\%)\end{array}$ & $\begin{array}{l}\text { Predicted range of drop } \\
\text { diameter from a force } \\
\text { balance } \\
(\mu \mathrm{m})\end{array}$ & $\begin{array}{l}\text { Predicted range of drop } \\
\text { diameter from a torque } \\
\text { balance } \\
(\mu \mathrm{m})\end{array}$ \\
\hline 1130 & 12.6 & 13.06 & 104.3 & 0.88 & 33 & 137 to 133 & 70 to 70 \\
\hline 1790 & 21.6 & 22.7 & 86.7 & 0.61 & 27 & 105 to 102 & 59 to 58 \\
\hline 2270 & 28.8 & 31.5 & 78 & 0.67 & 29 & 91 to 87 & 54 to 52 \\
\hline
\end{tabular}


Table 2 Median sunflower oil drop size as a function of shear stress using the modified Weissenberg rheometer at elevated continuous phase viscosity

\begin{tabular}{|c|c|c|c|c|c|c|c|}
\hline$\left(\mathrm{s}^{-1}\right)$ & $\begin{array}{l}\text { Shear stress } \\
\text { at start of } \\
\text { experiment } \\
\left(\text { dyne } / \mathrm{cm}^{2}\right) \\
\end{array}$ & $\begin{array}{l}\text { Shear stress } \\
\text { at end of } \\
\text { experiment } \\
\left(\text { dyne } / \mathrm{cm}^{2}\right)\end{array}$ & $\begin{array}{l}\text { Measured } \\
\text { median } \\
\text { diameter } \\
(\mu \mathrm{m})\end{array}$ & $\begin{array}{c}\text { Span of } \\
\text { distribution }\end{array}$ & $\begin{array}{l}\text { Coefficient of } \\
\text { variation } \\
(\%)\end{array}$ & $\begin{array}{l}\text { Predicted range of drop } \\
\text { diameter from a force } \\
\text { balance } \\
(\mu \mathrm{m})\end{array}$ & $\begin{array}{l}\text { Predicted range of drop } \\
\text { diameter from a torque } \\
\text { balance } \\
(\mu \mathrm{m})\end{array}$ \\
\hline 712 & 9.03 & 21.7 & 64.4 & 1.4 & 51 & 132 to 86 & 69 to 52 \\
\hline 1130 & 17.9 & 21.7 & 57.2 & 1.4 & 42 & 94 to 86 & 56 to 52 \\
\hline 1790 & 31.0 & 34.1 & 56.5 & 0.58 & 23 & 72 to 69 & 47 to 46 \\
\hline 2270 & 37.2 & 38.6 & 48.3 & 0.55 & 26 & 66 to 65 & 44 to 44 \\
\hline
\end{tabular}




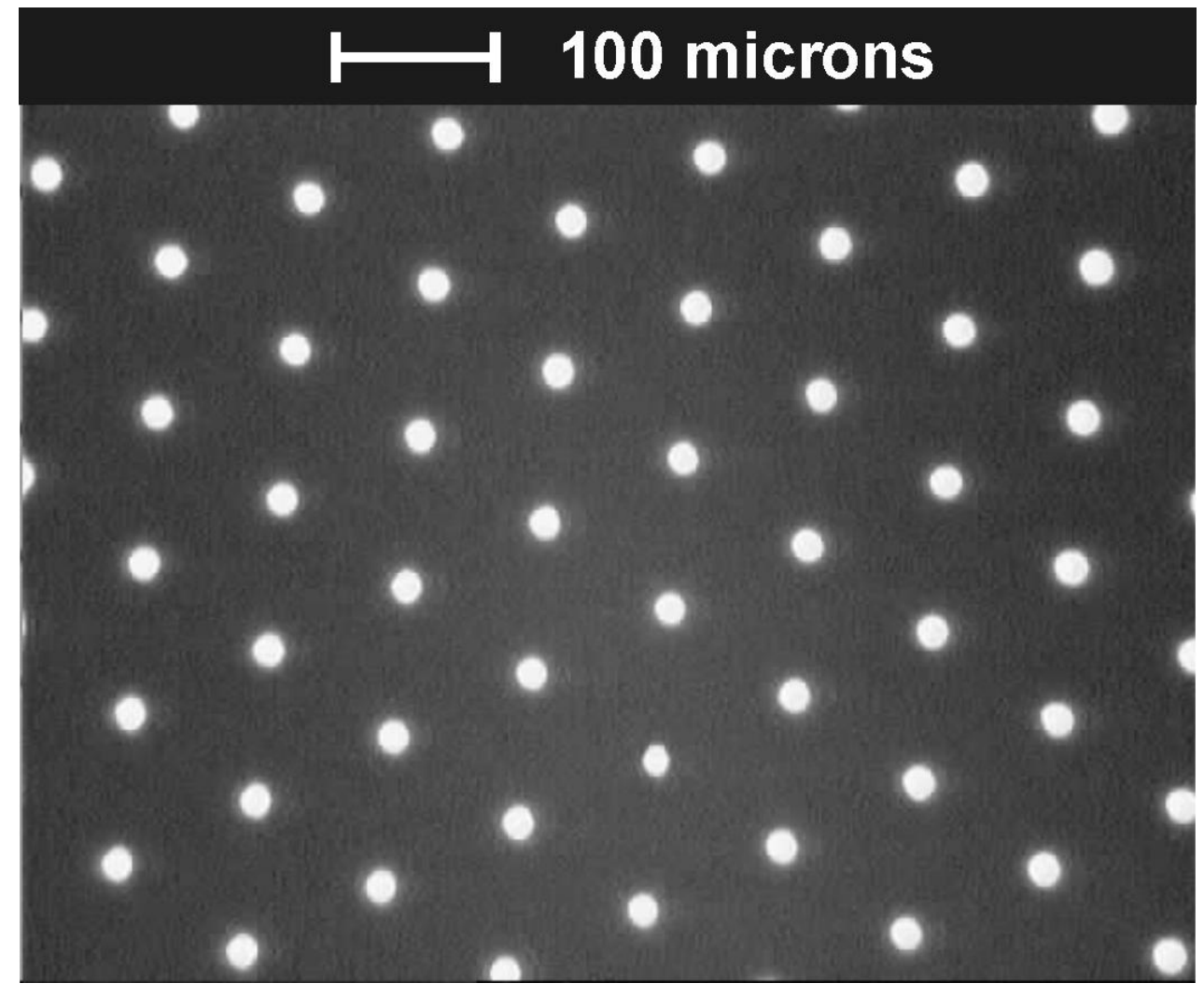

Figure 1 Regular pore array of a membrane used to produce dispersions 

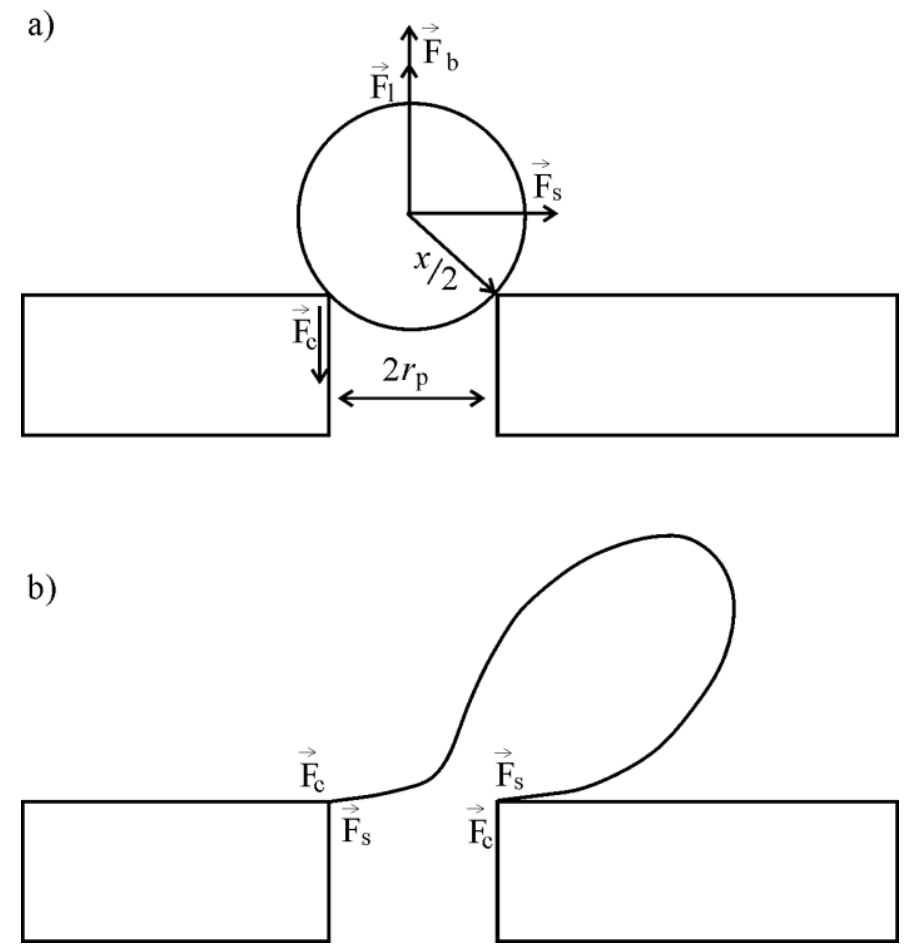

Figure 2 a) a weakly deformed droplet forming at the surface of a pore - leading to a torque balance at the centre of the drop and

b) a strongly deformed droplet - leading to a force balance at the membrane surface. 


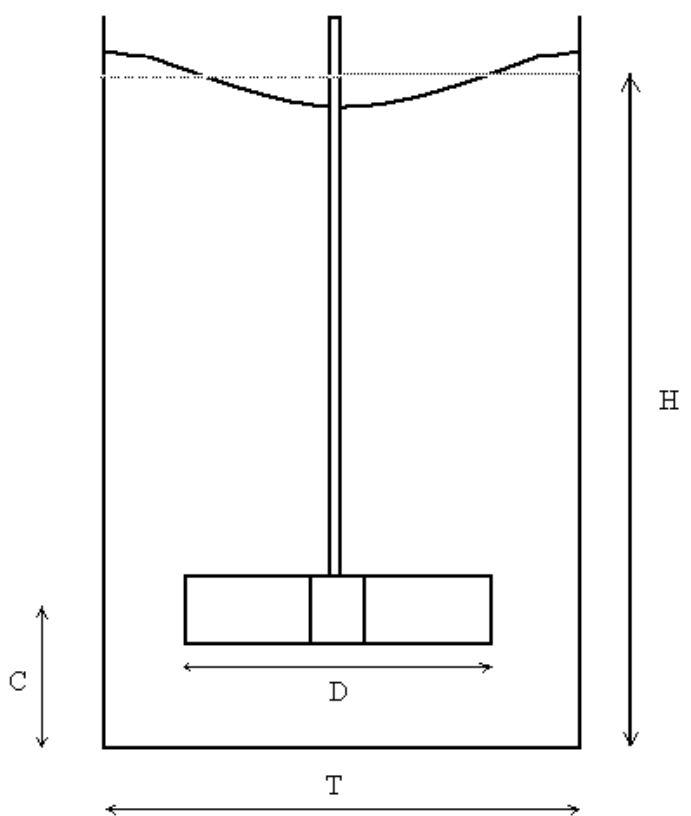

a)

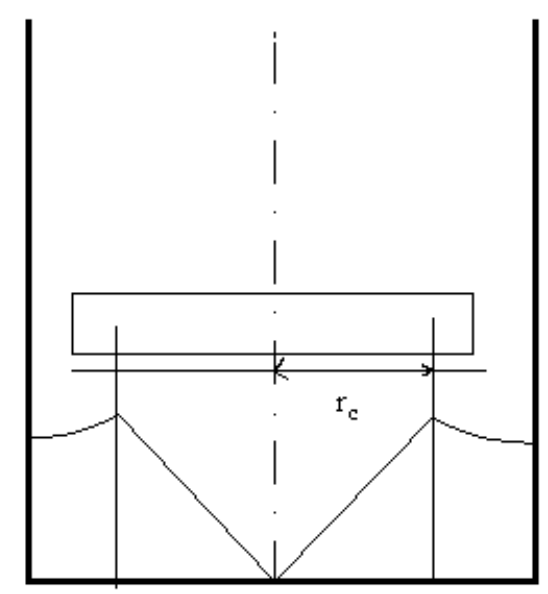

b)

Figure 3 a) Schematic of simple paddle blade stirred cell and

b) tangential velocity component under the impeller 


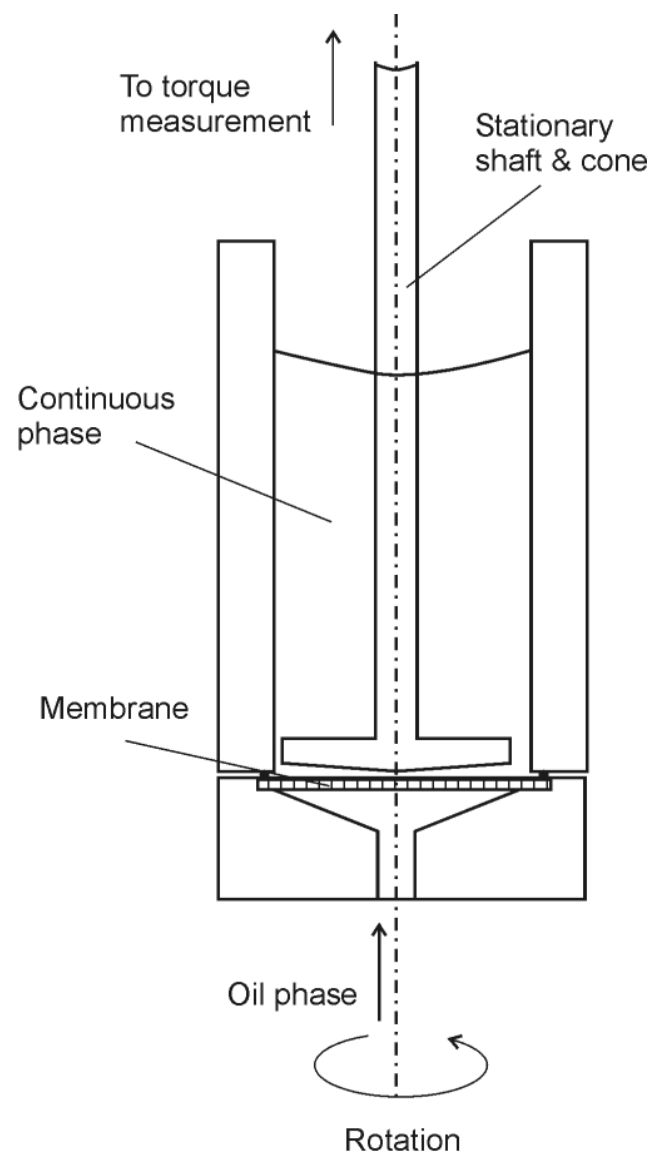

Figure 4 Modified Weissenberg rheometer for injection of oil in to aqueous phase 


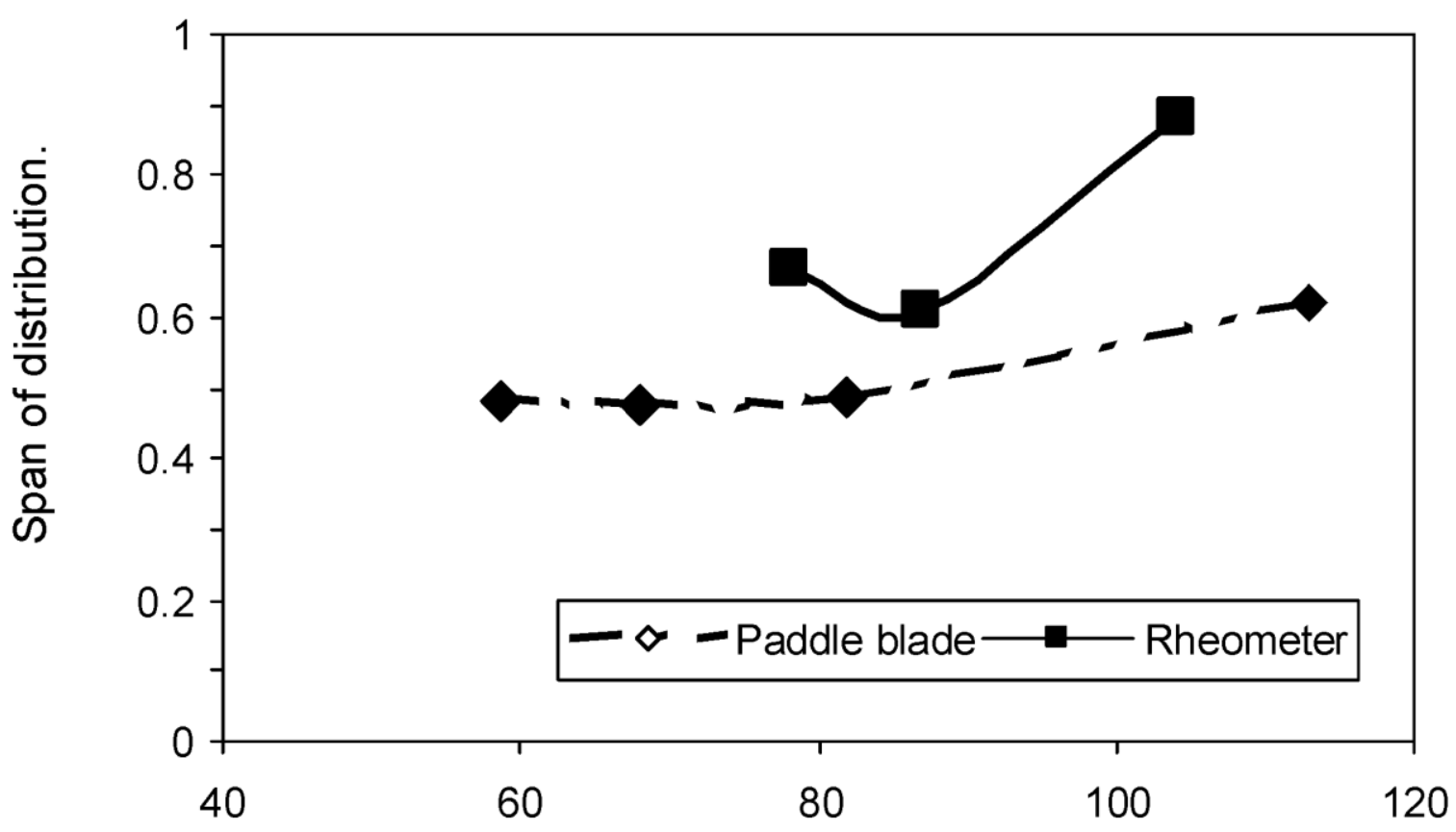

Median drop diameter, microns.

Figure 5 Comparison of drop size distribution spans produced using the two different devices for dispersion generation: the simple paddle blade stirred cell and the converted controlled shear rheometer 


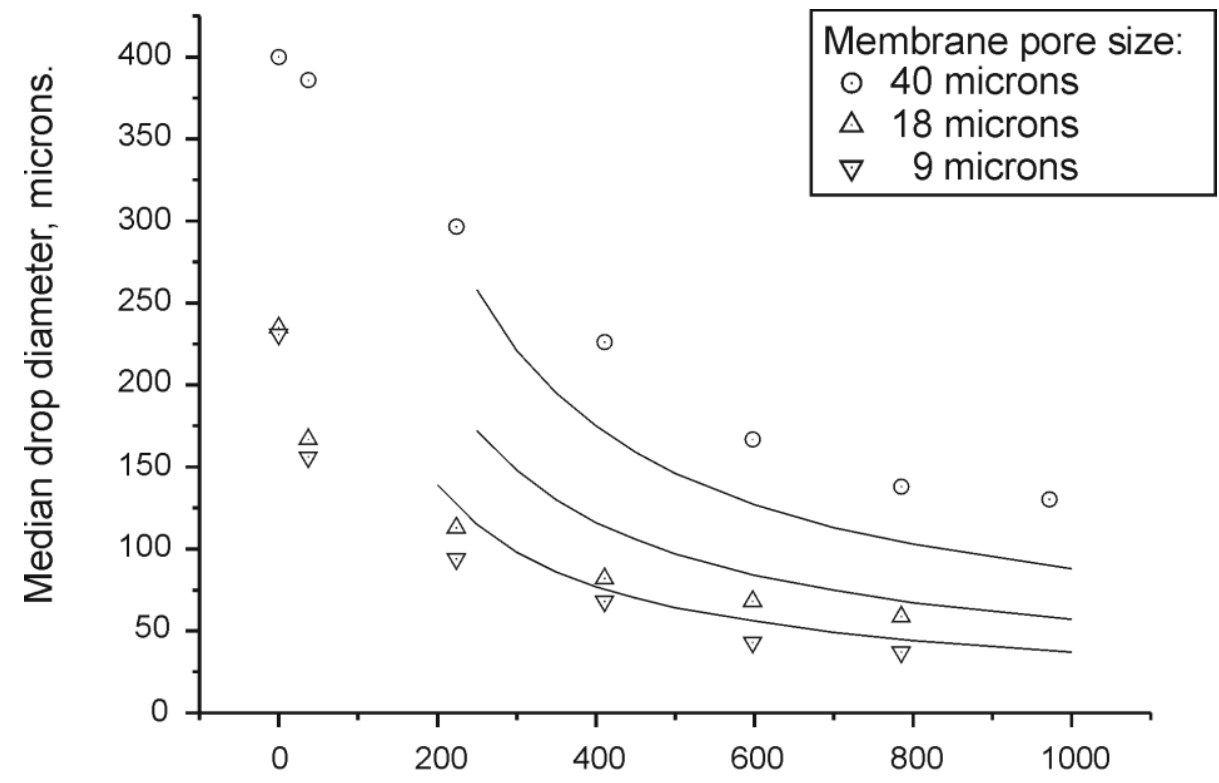

Paddle stirrer rotation speed, rpm.

Figure 6 Drop size against rotation speed for three different membranes sizes sunflower oil in water and $1 \%$ Tween 20 solution in the simple paddle blade cell: markers are data and curves are from equation (6) 


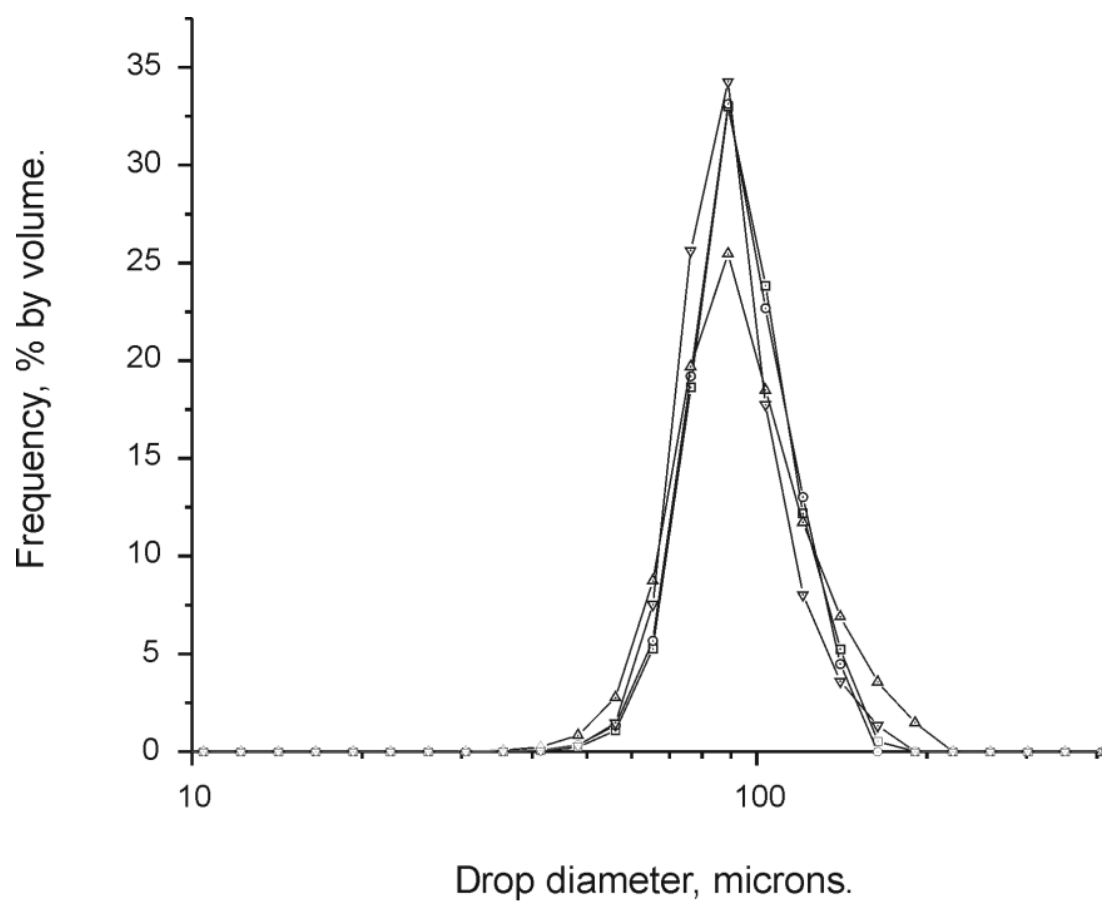

- - entire membrane open to oil injection flow

$-\odot-5 \mathrm{~mm}$ radius inactive at the center

$-\Delta-7.5 \mathrm{~mm}$ radius inactive at the center

$-\nabla-5 \mathrm{~mm}$ radius inactive at the center \& $5 \mathrm{~mm}$ strip at periphery

Figure 7 Drop size distributions for sunflower oil in water and 1\% Tween 20 solution in the small stirred cell using a $18 \mu \mathrm{m}$ membrane with different areas inactive with rotational speed $500 \mathrm{rpm}$ 


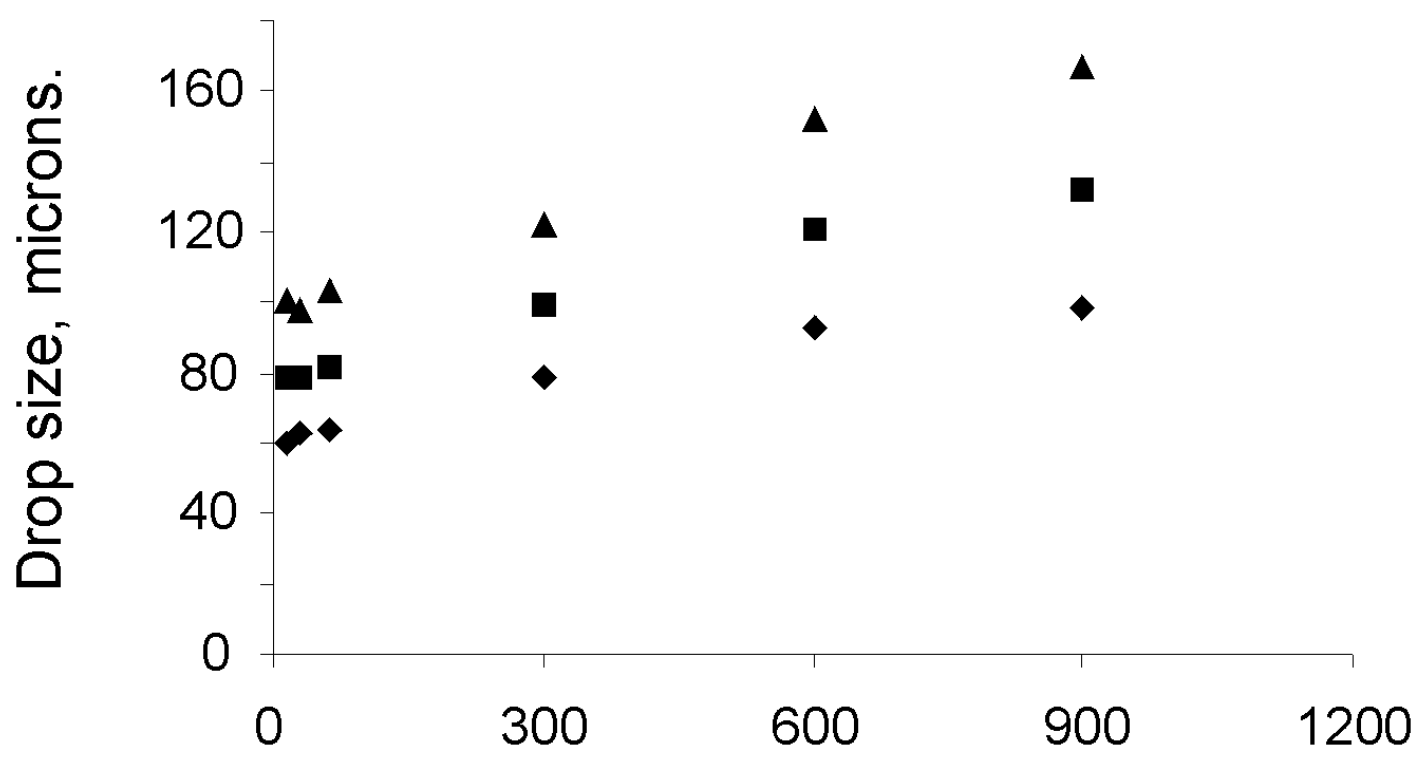

Specific oil injection rate, litres $\mathrm{m}^{-2} \mathrm{~h}^{-1}$.
$\diamond x_{01}$
- $x_{0.5}$
$\boldsymbol{\Delta} x_{0.9}$

Figure 8 Median drop sizes together with 10 and 90 percentile for sunflower oil in water and $1 \%$ Tween 20 solution in the small paddle blade stirred cell using an 18 $\mu \mathrm{m}$ membrane with various oil injection rates at a rotational speed $500 \mathrm{rpm}$ 